\title{
Anti-RSV Nanobody ALX-0171
}

National Cancer Institute

\section{Source}

National Cancer Institute. Anti-RSV Nanobody ALX-0171. NCI Thesaurus. Code C156058.

A trivalent nanobody composed of three monovalent $\mathrm{Nb017}$ moieties linked with glycineserine (GS) linkers, directed ag ainst the fusion protein (F protein) of human respiratory syncytial virus (RSV), with potential activity ag ainst RSV infections. Upon administration via nebulization and inhalation, the anti-RSV nanobody ALX-0171 targets and binds to RSV F protein, neutralizing the virus, and preventing viral entry into host cells. RSV F protein is a small envelope glycoprotein required for cytopathic syncytia formation that results from cell-to-cell fusion. 